\title{
Useful Life of Astrophysical Scientific Facilities
}

\section{Patricia Marquez, Oibar Martinez, Jose Miguel Miranda}

Department of Matter Structure, Thermal Physics and Electronics, Faculty of Physics, Complutense University of Madrid, Spain Email: patrmarq@ucm.es, oibarmar@ucm.es, miranda@ucm.es

How to cite this paper: Marquez, P., Martinez, O. and Miranda, J.M. (2021) Useful Life of Astrophysical Scientific Facilities. World Journal of Engineering and Technology, 9, 804-815.

https://doi.org/10.4236/wjet.2021.94056

Received: October 22, 2021

Accepted: November 7, 2021

Published: November 10, 2021

Copyright $\odot 2021$ by author(s) and Scientific Research Publishing Inc. This work is licensed under the Creative Commons Attribution International License (CC BY 4.0).

http://creativecommons.org/licenses/by/4.0/

\begin{abstract}
Large-scale astrophysical facilities have become increasingly relevant in certain key areas of scientific research but typically require strong financial investments. It is, therefore, crucial to gain a deep understanding of what could be a foreseeable lifespan of a given instrument before providing the required fund to build it. In this paper, we intend to contribute to this understanding with a study of the lifespan of past, current and future observatories and telescopes. The methodology has been based on the compilation of relevant data from twenty telescopes, three of them mounted on space satellites and the other seventeen distributed worldwide. An analysis of the main limiting factors that affect the lifetime of an astrophysical facility is also presented.
\end{abstract}

\section{Keywords}

Lifespan, Obsolescence, Astrophysical Instruments, Telescopes

\section{Introduction}

Since the 16th and 17th centuries, with the first optical telescope built by Galileo and observations of Kepler and Copernicus, the development of instruments and technologies (photography, spectroscopy and photometry) has increased notably [1]. At the present, there are large installations around the world for researching the universe in addition to space telescopes [2] and these installations and space telescopes have a lifespan.

An astrophysical research infrastructure can provide a number of economical and social benefits such as base knowledge, technological advances, human capital formation, cultural effects and services to third parties, to name a few. However, it also demands a large amount of human effort and financial investment.

Despite the intuitive concept that a general reader has of the term "lifespan", 
the lifespan of research infrastructure is not a parameter that can be found formally defined in any international standard. However, the lifespan is a key factor to assess the cost-benefit balance of any large-scale investment.

It is a common practice to define specific requirements for the operational lifetime of a research infrastructure during the design phase, but this information is usually distributed in documents with limited access.

In this paper, we show a compilation of available information on the ageing and reliability of twenty astrophysical instruments, together with an analysis of the main limiting factors that affect the lifetime of an astrophysical facility.

\section{Methods and Results}

We have gathered and organized relevant data on operational lifetime and reliability challenges from twenty telescopes, three of them mounted on space satellites and the other seventeen distributed worldwide and covering microwave, infrared, visible and gamma ray radiation measurements.

As far as satellite instrumentation concerns, NASA suggests that the lifespan of a space telescope be 15 years [2]. One space telescope that has gotten important astronomical discoveries is the Hubble Space Telescope. The Hubble Space Telescope was put in orbit on 24th April 1990. This telescope was designed to be maintained and upgrated periodically by astronauts in space. The Hubble Space Telescope was designed with modular pieces to facilite maintenance. It permitted to complete missions from 1993 to 2009 [2]. The Hubble Space Telescope had an estimated lifespan of 15 years, nevertheless, 30 years after its launch, it continues working and taking data.

The Gamma-Ray Fermi Telescope started to operate in 2008. Fermi has two instruments, the Large Area Telescope (LAT) and the Gamma-ray Burst Monitor (GBM). The initial lifespan of Fermi was five years, but it has been extended and Fermi is still in operation [3] [4].

The James Webb Space Telescope (JWST or Webb) will be a space instrument with a large infrared telescope with a 6.5 metre primary mirror. The mission lifespan is greater than 10 years but, in this case, the limitation is the fuel used to maintain it in orbit. The telescope will be put in orbit in 2021 [5] [6].

The ground-based telescopes are cheaper than space telescopes and can obtain very good quality information about space. The maintenance and upgrades are easier and to operate with them is more accessible [7]. Figure 1 shows the electromagnetic spectrum. We can find different types of ground-based telescopes, depending on the range of the operating frequencies radio telescopes, microwave, gamma-ray, solar, optical and infrared telescopes [8]. The ground-based gamma-ray telescopes operate above a few $10 \mathrm{GeV}$ energy threshold, which is considered as very-high energy range (VHE), in contrast to high energies (HEs, $\mathrm{MeV}-\mathrm{GeV}$ gamma rays), at which space-based gamma-ray detectors operate [8].

These are some of the most important ground-bases telescopes around the world. 


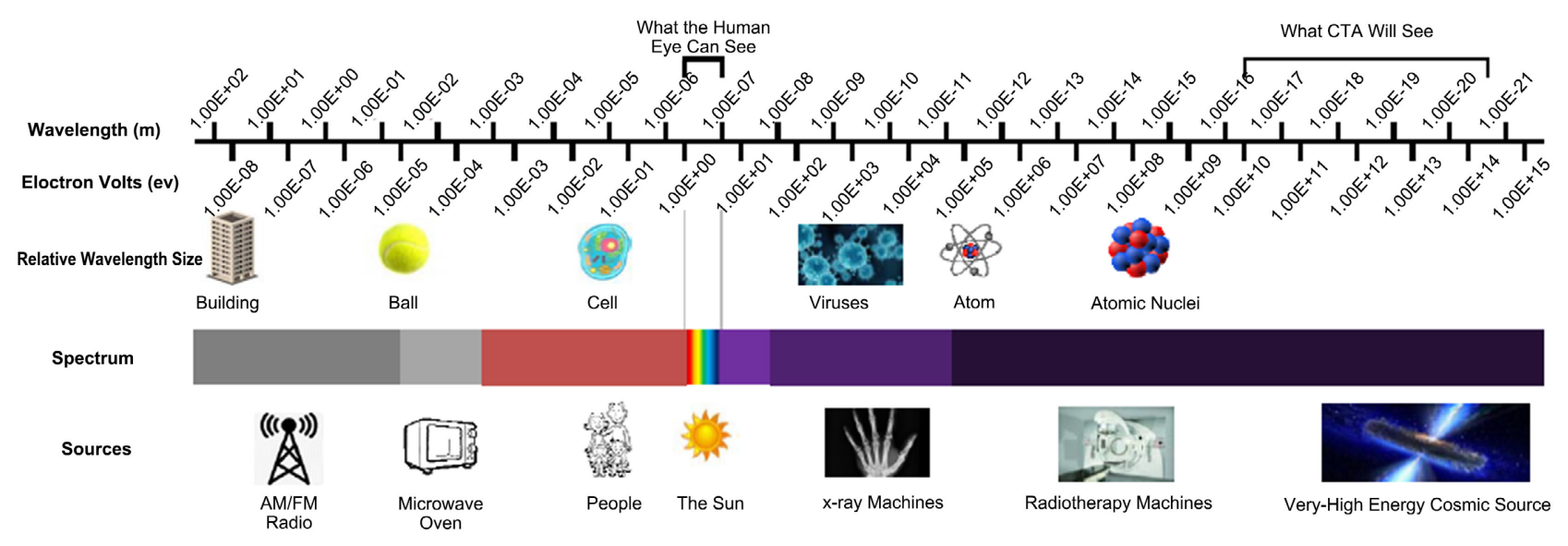

Figure 1. Electromagnetic spectrum based on [9].

- The Swedish Solar Telescope (SST) in the Observatorio Roque de Los Muchachos (ORM) is the largest solar telescope in Europe. The lens is made of fused silica and it is $1 \mathrm{~m}$ diameter. The resolution of this telescope is $0.1 \mathrm{arc}$ seconds in blue light, this corresponds to $70 \mathrm{~km}$ distance on the solar surface [10]. This telescope is sheltered in a building. The first data were taken in 2002.

- European Solar Telescope (EST): The installation of the EST telescope in the ORM was approved on May 2021. It is a telescope with a 4.2 metre primary mirror and it will study the magnetic coupling of the solar atmosphere. The construction will start in 2023/2024 and it will continue during 5 years. The Operation Phase is expected to last 30 years [11].

- The Gran Telescopio de Canarias (GTC) is an optical telescope. The primary mirror is made of "Zerodur" and it is 10.4 metres of diameter. It is one of the most advanced telescopes in the world and the largest of the optical infrared ones. There are a secondary and a tertiary mirror too. With the primary mirror they produce the telescope focal plane in the focal station of choice. The telescope is mounted in a steel structure, which allows the rotational movements of the telescope. The telescope is in a building with a dome that protects it from environmental hazards. The dome was designed to minimize the turbulence of the air close to the telescope. The expected lifespan of GTC is 50 years [12] [13] [14].

- Thirty Meter Telescope (TMT): the TMT would be one of the most advanced and powerful ground-based telescope and the largest optical/nearinfrared telescope in the northern hemisphere [15]. The ORM is a candidate location for the construction of this telescope. The lifespan of TMT will be 50 years [16].

- The Very Large Telescope (VLT) is a group of 4 optical telescopes with mirrors of $8.2 \mathrm{~m}$ in diameter. It is installed in the Observatory of Cerro Paranal in Chile. The first light was in 2000 and the nights of observation are a lot because the location does not have luminal pollution and the desert climate is favourable [17]. 
- The Extremely Large Telescope (ELT) will be a new optical telescope and it will be in the desert of Atacama, in Chile. The operation time will be around 30 years. The telescope will be in a building with a dome due to the extreme environment of this location. The construction will start in 2027 [18].

- The Gemini Observatory (GO) is an Observatory of 2 identical telescopes, one in Mauna Kea (Hawaii) and the other in Cerro Pachon (Chile). It is an optical and infrared telescope. The first light was in 1999 and 2000, respectively. They are in two good locations for observing the entire sky [19].

- High Energy Stereoscopic System (H.E.S.S.) is an array of Imaging Atmospheric Cherenkov Telescopes (IACTs) that investigates cosmic gamma rays in the energy range from $10 \mathrm{~s}$ of $\mathrm{GeV}$ to $10 \mathrm{~s}$ of $\mathrm{TeV}$. Scientists can investigate the gamma-ray sources with intensities at a level of a few thousandths of the flux of the Crab nebula ${ }^{1}$. H.E.S.S. is located in Namibia. The first of the four $12 \mathrm{~m}$ telescopes of the Phase I of H.E.S.S. started the operation in 2002, all four were operating in December 2003 and they were officially inaugurated in September 2004. The fifth ( $28 \mathrm{~m}$ large) telescope (H.E.S.S.-II) was inaugurated in 2012. In 2016 the cameras of the four H.E.S.S.-I telescopes were fully refurbished using the new electronics designed for the next generation IACT array, CTA [20].

- The Whipple Telescope (WT) was a previous generation IACT, which made first detections of gamma-ray astrophysical sources in the VHE range. It was located at the Fred Lawrence Whipple Observatory, in Arizona. It was taking data since 1968 until 2013 [21].

- The Very Energetic Radiation Imaging Telescope Array System (VERITAS) is a ground-based gamma-ray instrument operating at the Fred Lawrence Whipple Observatory (FLWO) in southern Arizona, USA. It is an array of four $12 \mathrm{~m}$ IACTs for gamma-ray astronomy in the GeV-TeV energy range [22].

- The project of Major Atmospheric Gamma Imaging Cherenkov (MAGIC) telescopes started in 1996. They are two telescopes of $17 \mathrm{~m}$ in diameter. The first one, MAGIC I, started operations in 2004 and the second one, MAGIC II, started in 2009. Since 2009 MAGIC I and MAGIC II operate in coincidence in the stereoscopic mode. These telescopes are of IACT type and they detect gamma rays of very-high energy. The telescopes are not in a building, and then they are exposed to environmental hazards [23] [24]. The lifespan of this type of experiments is around 10 years [25]. MAGIC I and MAGIC II are in operation by more than 10 years and are together with H.E.S.S. and VERITAS IACT arrays the three most sensitive VHE astrophysical gamma-ray detectors nowadays.

- The Cherenkov Telescope Array (CTA) Observatory will be next generation full-sky (with two sites, the Sourthern one in Paranal, Chile, the Nothern one in La Palma, Spain) VHE gamma-ray facility, with an order of magni-

${ }^{1}$ The Crab Nebula is the brightest steady source of gamma rays in the sky. 
tude better gamma-ray flux sensitivity in a large $20 \mathrm{GeV}$ to $300 \mathrm{TeV}$ energy range with respect to current IACT arrays. To reach this goal, 10s of IACTs will be deployed, which will provide the improvement of angular and energy resolution with respect to nowadays instruments as well. As a result, the number of detected VHE gamma-ray astrophysical sources will increase considerably, from about 280 today [26] to more than 1000 [27] [28]. The operation of CTA will have an estimated duration of 30 years [9] [29].

- The High Energy Gamma Ray Astronomy (HEGRA) was an imaging atmospheric Cherenkov telescope array for VHE Gamma-ray astronomy that was taking data since 1987 till 2002 [30] when it was removed to install the project MAGIC.

- The radio telescope in the Observatory of Arecibo, in Puerto Rico, is a fixed telescope and the observation is limited by the position of the Earth. The reflector diameter is $305 \mathrm{~m}$. It is surrounded by four mountains. This telescope had the first observation on 1st November 1963.

On 10th August 2020 one cable of 8 centimetre thick steel fell and it cut the plate of the telescope, the initial damage was in the anchor point of the cable. Theoretically the lifespan of this structure is 50 years but it was upgraded in 1997 with the installation of the new dome [31]. 3 months later, on 6th November, a second cable fell too. In that moment, the engineers were planning the reparation of the first cable. On 1st December, more cables broke and the structure collapsed. This telescope survived tropical storms, but the Isaias tropical storm was in July 2020 and it is not clear if the damage of the first cable was due to this storm [32]. There were questions whether the cables were maintained correctly [33] but the reason of the collapse is not clear yet, one hypothesis is the adverse meteorological events.

- The largest radio-astronomy array is the Very Large Array (VLA) in Nuevo Mexico (USA). This array has 28 antennas. The formal inauguration of the VLA started in 1980 [34]. The construction lasted 10 years [35].

- The Atacama Large Millimetre/submillimetre Array (ALMA) is a microwave telescope. It is in Atacama desert, Chile, at 5000 metres above sea level [36]. The lifespan of ALMA is at least 30 years. The first light was in September 2011. ALMA is an observatory with 64 antennas of $12 \mathrm{~m}$ diameter [37]. The location of astrophysical scientific facilities can be seen in Figure 2 .

\section{Discussion}

Figure 3 shows a general overview of the lifespan of previously described telescopes. Unfortunately, the details of the estimations of the lifespan of these facilities are difficult to find and are often confidential.

According to data provided in previous section, we can conclude that the lifespan of space telescopes is estimated in 15 years. By default, no maintenance (for hardware items) can be performed for space telescopes. However, the lifespan value can experience a drastic reduction if the instrument has limitations in terms of fuel storage. 


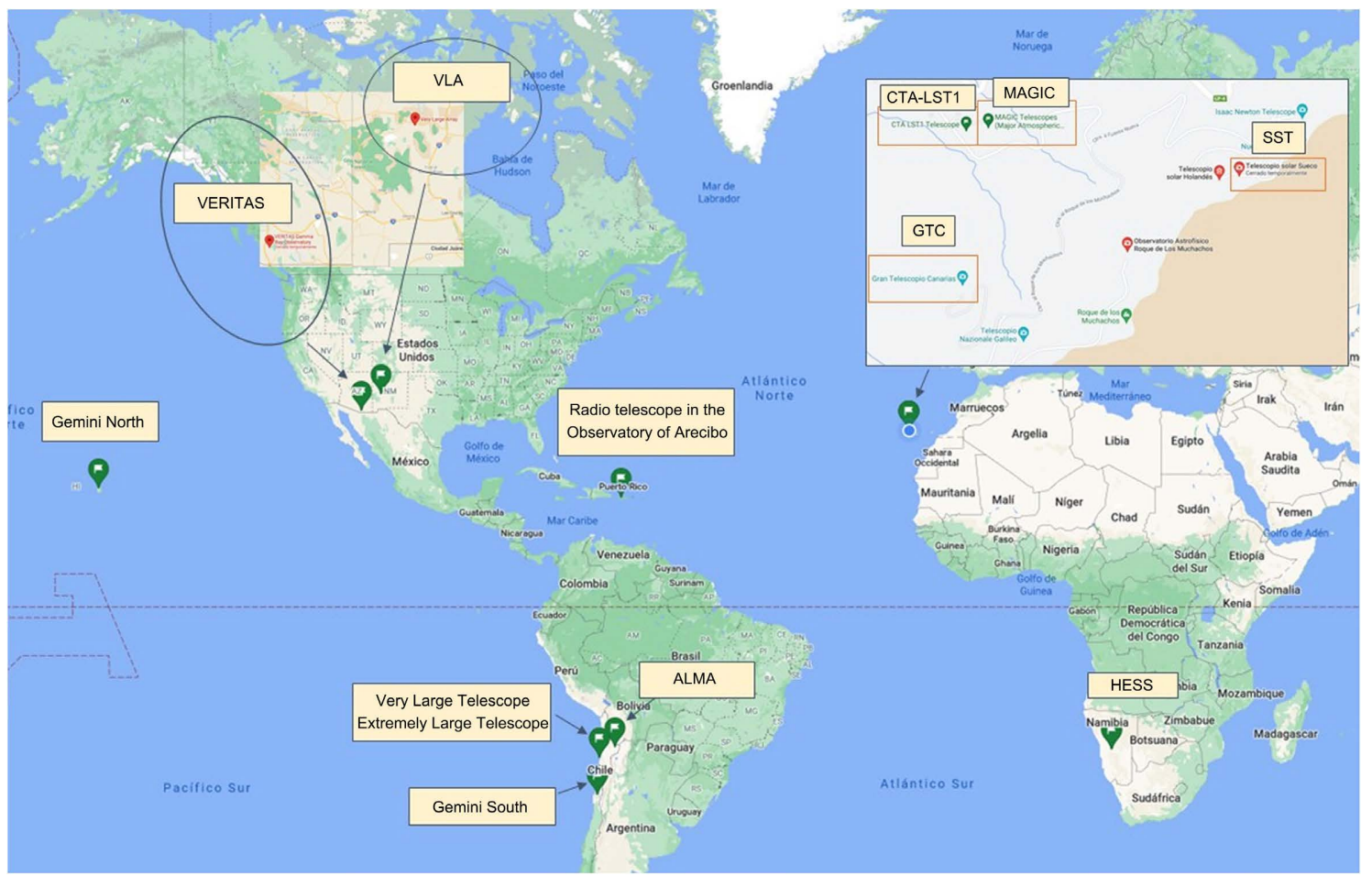

Figure 2. Location of astrophysical scientific facilities.

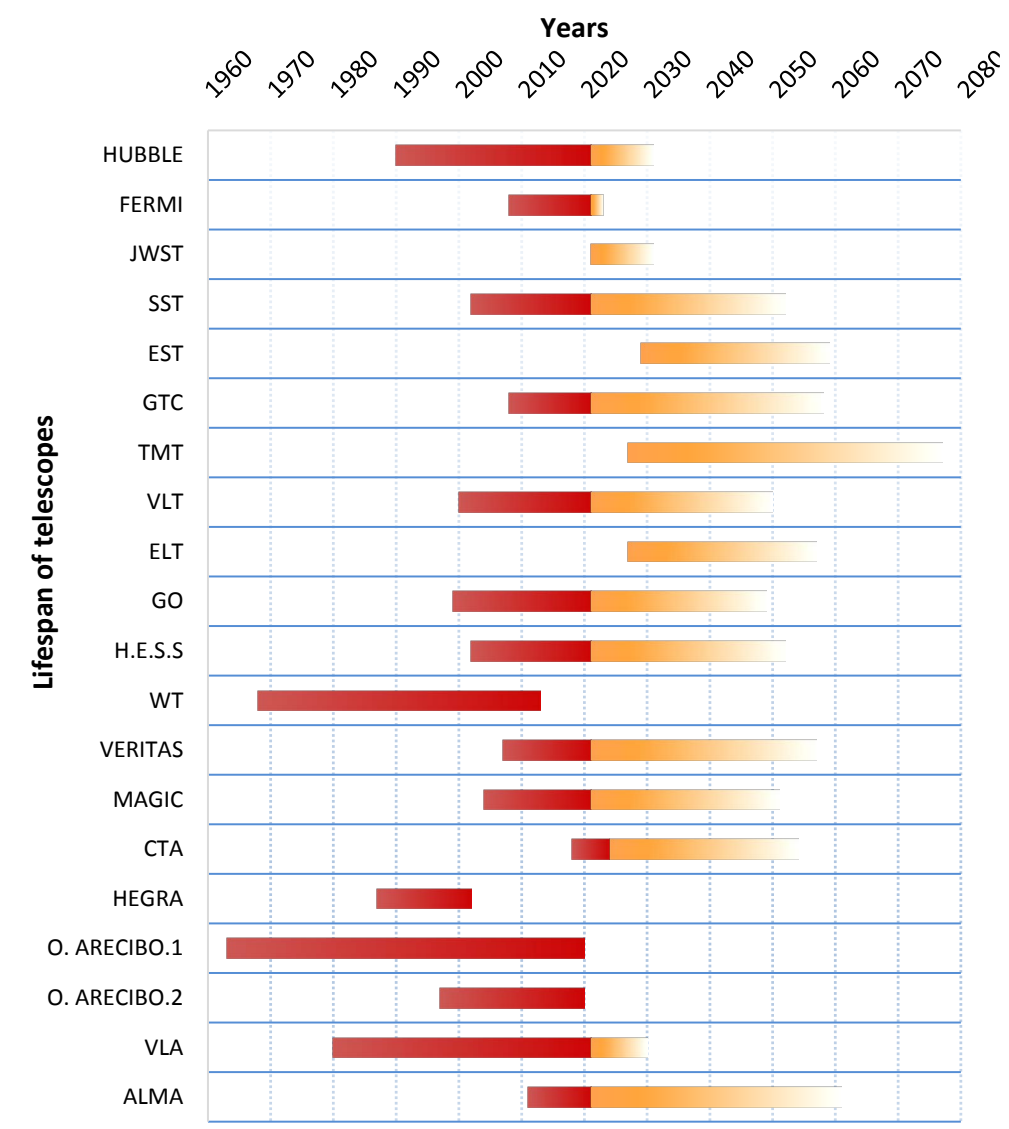

Figure 3. Lifespan of telescopes listed in the paper (red: current lifespan, orange: expected future lifespan). Note: The operation of CTA will start after commissioning phase. 
In addition, it is also concluded that the ground-based telescopes have an estimated lifespan between 10 and 50 years. Concerning solar telescopes, it was possible to obtain information on the lifespan from only one of them, which is estimated in 30 years. The lifespan of those optical telescopes where the necessary information was found is estimated in 50 years. The initial lifespan of IACT telescopes is estimated in around 10 years, but they use to operate for significantly longer times. No information on the lifespan of radio telescopes and microware telescopes was available.

Despite the large variety of designs that one can encounter for ground based telescopes, all of them share a common feature: they must be mounted on a concrete foundation which uses common materials. The lifespan of the concrete structures is defined during the design of the structure according to the local regulations in terms of civil engineering that currently apply to the site.

In the case of Spain (Observatory Roque de Los Muchachos), the lifespan of the concrete is defined in the Structural Concrete instruction (EHE-08) (Real Decreto 1247/2008, of 18th of July) [38]. The Table 1 is from the chapter 01 of this document. In the case of scientific facilities, the design working life is 50 years. For scientific facilities in Spain without any information about the lifespan, a lifespan of 50 years has been estimated.

The BS EN 1990 Eurocode is the standard approved in Europe for the definition of the working lifespan on the concrete structure [39]. Table 2 shows the working life of concrete structures according to this standard. The category corresponding to scientific facilities would be four, and therefore the corresponding working life should be 50 years.

In United States, the American Concrete institute defines the requirements of concrete structures. The standard that defines these requirements is ACI 318.

Table 1. Design working life of the various types of structure [38].

\begin{tabular}{lc}
\hline \multicolumn{1}{c}{ Type of structure } & Design working life \\
\hline $\begin{array}{l}\text { Structures of a temporary nature } \\
\begin{array}{l}\text { Replaceable elements not forming part of the main structure } \\
\text { (for example, handrails, pipe supports) }\end{array}\end{array}$ & Between 3 and 10 years \\
$\begin{array}{l}\text { Agricultural or industrial buildings (or installations) and } 25 \text { years } \\
\text { maritime works }\end{array}$ & Between 15 and 50 years \\
$\begin{array}{l}\text { Housing or office buildings, bridges or crossings of a total } \\
\text { length of less than } 10 \text { metres and civil engineering structures } \\
\text { (except maritime works) having a low or average economic }\end{array}$ & \\
repercussion & 50 years \\
$\begin{array}{l}\text { Buildings of a monumental nature or having a special } \\
\text { importance }\end{array}$ & 100 years \\
$\begin{array}{l}\text { Bridges of a total length equal to or greater than } 10 \text { metres } \\
\text { and other civil engineering structures having a high economic } \\
\text { repercussion }\end{array}$ & 100 years
\end{tabular}


Table 2. Indicative design working life according to the European standard [39].

\begin{tabular}{|c|c|c|}
\hline $\begin{array}{c}\text { Design } \\
\text { working life } \\
\text { category }\end{array}$ & $\begin{array}{c}\text { Indicative } \\
\text { design working } \\
\text { life (years) }\end{array}$ & Examples \\
\hline 1 & 10 & Temporary structures \\
\hline 2 & 10 to 25 & Replaceable structural parts, e.g. gantry girders, bearings \\
\hline 3 & 15 to 30 & Agricultural and similar structures \\
\hline 4 & 50 & Building structures and other common structures \\
\hline 5 & 100 & $\begin{array}{l}\text { Monumental building structures, bridges and other civil } \\
\text { engineering structures. }\end{array}$ \\
\hline
\end{tabular}

According to this standard, "ACI 318 addresses serviceability through strength requirements and limitations on service load conditions" [40]. As a result, this standard does not enable one a straightforward estimation of a scientific facility lifespan.

Maintenance activities during the instrument lifespan are needed to ensure the integrity of the structure. A maintenance plan needs to be implemented in order to organise and coordinate all the maintenance activities needed to ensure the proper operation of the instrument. The inspections must be done by qualified staff. It is customary to make use of these inspections to proceed with any non-regular maintenance actions that might be needed. The critical damages depend of the type of structure and the location, e.g. a wide crack in the reinforcement concrete near to sea can be very dangerous because the corrosion in maritime ambience is faster than in non-maritime one and if it arrives to the reinforcement it can expand and produce the collapse of the structure. In this case, the crack must be sealed as soon as possible and if the reinforcement is damaged, it must be repaired. The international standards [41] [42] provide guidelines to identify the maximum acceptable width of cracks.

In practice, the real lifespan of an instrument is usually smaller than the lifespan of the structure. This may be due to the following reasons:

$\checkmark$ Obsolescence: there are four types of obsolescence [43] that can affect the lifespan of the scientific facility:

- Logistical: the pieces (materials, software or manufacturing) can not be procured

- Functional: the system or subsystem is still working but the requirements have changed, then, the system or subsystem is obsolete because the function, performance or reliability are different.

- Technological: there are advanced components every day. This can be a problem if spare parts need to be bought from the manufacturer, since only more advanced components could be available

- Functionality Improvement Dominated Obsolescence (FIDO): the manufacturer must change the products for maintaining the market competition with another similar manufacturer. 
The four types of obsolescence can affect only one part of the facility. If the obsolescence of a part of the facility conditions the lifespan of it, an evaluation about the opportunity to change this part, needs to be done.

- Catastrophic events: the scientific facilities are designed according to environmental specifications. These specifications are established according to the historical information of the place where the facility will be built. When there is an extraordinary environmental event, which is stronger than the specifications, the facility can suffer extreme consequences. The damage evaluation after the event versus the cost of the reparation is what decides if the operation life of the facility continues or not. A catastrophic event can happen in any moment.

- Lack of financial support for the maintenance: normally the scientific facilities are public projects and the financial support is linked to public budgets. The public budgets are approved yearly. When a scientific facility is going to be built, there is a compromise with the public authorities for operating and maintaining the facility $\mathrm{X}$ years. However, if there are setbacks in the economy (e.g. economic crisis), the public budgets can be affected, then, also the scientific projects. In USA, Spain and other countries there have been cuts in scientific domain in the last years [44] [45] but it is difficult to find references about that.

- Misuse of the instrument: during the operation phase, the operators of the scientific facilities must follow the operation manual of the facility. If the manual is not followed correctly, the facility can be damaged by a misuse of the instrument. In addition to operation manual, the operators must have a formation according to the activities.

\section{Conclusions}

Scientific facilities are very expensive but they cannot be used indefinitely.

Two major factors limit the lifespan of space telescopes: maintenance difficulties and fuel consumption. Maximum operation time in ground telescopes is limited by the structure. The lifespan of the structure of ground-based telescopes is usually longer than the lifespan of the whole telescope because there are several factors that affect the real life during the operation phase of the facility. For having a long real lifespan of the facilities, a constant balance between the cost of the installation and the benefits of the operation is needed. A proper spare and maintenance program and trained operators are needed to keep the facilities in good conditions.

Every installation has lifespan limitations depending on specific reliability and maintenance issues. It is therefore compulsory to implement and complete a detailed reliability, availability and maintainability plan matched to the specific needs of the infrastructure.

\section{Acknowledgements}

This work has been partially funded by the Spanish Ministry of Science and Educa- 
tion, via "Retos de investigación" programme, project id. PID2019-107847RB-C43.

Authors are thankful to the CTA LST project for sharing the technical information about the project.

\section{Conflicts of Interest}

The authors declare no conflicts of interest regarding the publication of this paper.

\section{References}

[1] Alberdi, A. (2021) Large Astronomical Facilities: Astrophysics on a Grand Scale. Lychnos, 9. http://www.fgcsic.es/lychnos/en_en/articles/large_astronomical_facilities

[2] NASA (2021) About-The Hubble Story. https://www.nasa.gov/content/about-the-hubble-story

[3] Dooling, D. (2019) Fermi Gamma-Ray Space Telescope. Encyclopedia Britannica. https://www.britannica.com/topic/Fermi-Gamma-ray-Space-Telescope

[4] NASA (2021) Fermi Gamma-Ray Space Telescope Website. Nasa Website. https://www.nasa.gov/content/fermi-gamma-ray-space-telescope

[5] NASA (2018) James Webb Telescope Overview. https://www.nasa.gov/mission_pages/webb/about/index.html

[6] NASA (2021) James Webb Space Telescope. Frequently Asked Questions. https://jwst.nasa.gov/content/about/faqs/faq.html

[7] Beck, K. (2017) What Advantages Do Space Telescopes Have over Telescopes Used on Earth? sciencing.com. https://sciencing.com/infrared-telescope-work-4926827.html

[8] Cuesta, L. and Ruiz, N. (2005) The World's Big Telescopes and Astronomy Paradises. Ars Medica. Revista de Humanidades, 4, 58-74.

http://www.dendramedica.es/revista/v4n1/grandes_telescopios_del_mundo_y_parai sos_astronomicos.pdf

[9] CTA Consortium (2021) Official Website: Cherenkov Telescope Array (CTA). https://www.cta-observatory.org/project/technology/lst

[10] Scharmer, G.B., Bjelksjo, K., Korhonen, T.K., Lindberg, B. and Petterson, B. (2002) The 1-Meter Swedish Solar Telescope. Proceedings of Innovative Telescopes and Instrumentation for Solar Astrophysics, 4853, 10. https://doi.org/10.1117/12.460377

[11] EST Consortium (2021) Official Website: EST. https://www.est-east.eu

[12] Rodríguez, L.A. (2020) Progress on GTC Engineering Operations. GTC Users Committee, 12 .

[13] Gran Telescopio de Canarias (2021) Official Website: Gran Telescopio CANARIAS. http://www.gtc.iac.es

[14] Perez, R. (2020) El Grantecan cambiará sus investigaciones si el TMT llega a la Isla. https://www.eldia.es/la-palma/2020/10/25/grantecan-cambiara-investigaciones-tmt$\underline{\text { lega-22314993.html }}$

[15] TMT International Observatory LLC (2021) Official Website: TMT La Palma. http://tmtlapalma.org/en/about-us

[16] Gabriel Henríquez Pérez SLP (2017) Documento Inicial del Proyecto: Thirty Meter Telescope. 
https://www.puntagorda.es/wp-content/uploads/2017/05/TMT-Documento-Inicial_ 24.04.17.pdf

[17] European Southern Observatory. The Very Large Telescope. The World's Most Advanced Visible-Light Astronomical Observatory. https://www.eso.org/public/archives/handouts/pdf/handout_0003.pdf

[18] European Southern Observatory (2021) Official Website: ELT. https://www.eso.org

[19] Gemini Collaboration (2021) Official Website: Gemini Observatory. https://www.gemini.edu

[20] H.E.S.S. Collaboration (2021) Official Website: H.E.S.S. The High Energy Stereoscopic System. https://www.mpi-hd.mpg.de/hfm/HESS

[21] Kildea, J., et al. (2007) The Whipple Observatory 10 m Gamma-Ray Telescope, 19972006. Astroparticle Physics, 28, 182-195.

https://doi.org/10.1016/j.astropartphys.2007.05.004

[22] VERITAS Collaboration (2021) Official Website: VERITAS. https://veritas.sao.arizona.edu

[23] Tescaro, D. (2014) The Upgraded MAGIC Cherenkov Telescopes. Nuclear Instruments and Methods in Physics Research Section A: Accelerators, Spectrometers, Detectors and Associated Equipment, 766, 65-68. https://doi.org/10.1016/j.nima.2014.04.037

[24] Aleksić, J., et al. (2016) The Major Upgrade of the MAGIC Telescopes, Part I: The Hardware Improvements and the Commissioning of the System. Astroparticle Physics, 72, 61-75. https://doi.org/10.1016/j.astropartphys.2015.04.004

[25] de Naurois, M. and Mazin, D. (2015) Ground-Based Detectors in Very-High-Energy Gamma-Ray Astronomy. Comptes Rendus Physique, 16, 610-627.

https://doi.org/10.1016/j.crhy.2015.08.011

[26] Wakely, S. and Horan, D. (2021) TeVCat Online Catalog. http://tevcat.uchicago.edu

[27] The CTA Consortium, et al. (2019, September 22) Science with the Cherenkov Telescope Array. https://www.worldscientific.com/worldscibooks/10.1142/10986

[28] De Almeida, B., et al. (2014) The Large Size Telescope of the Cherenkov Telescope Array. Proceedings of SPIE, 9145, 91450-91451. https://doi.org/10.1117/12.2054605

[29] CTA Consortium (2021) CTA JAMA Data Base. https://jama.cta-observatory.org

[30] MPI (2021) The HEGRA Experiment WEBSITE. https://www.mpi-hd.mpg.de/hfm/HEGRA/HEGRA.html

[31] Clery, D. (2021) Fallen Giant. Science (80-.), 371, 225-229. https://doi.org/10.1126/science.371.6526.225

[32] Clery, D. (2021) How the Famed Arecibo Telescope Fell-And How It Might Rise Again. sciencemag.org.

https://www.sciencemag.org/news/2021/01/how-famed-arecibo-telescope-fell-andhow-it-might-rise-again

[33] Witze, A. (2020) Gut-Wrenching Footage Documents Arecibo Telescope's Collapse. Nature. https://doi.org/10.1038/d41586-020-03421-y

[34] Mann, A. (2020) The Very Large Array: 40 Years of Groundbreaking Radio Astronomy. space.com. https://www.space.com/very-large-array.html

[35] Kellermann, K.I., Bouton, E.N. and Brandt, S.S. (2020) The Very Large Array. In: Kellermann, K.I., Bouton, E.N. and Brandt, S.S., Eds., Open Skies, Springer, Cham, 319-390. https://doi.org/10.1007/978-3-030-32345-5_7

[36] European Commission (2013) ALMA Observatory Opens Window to Universe's 
Darkest Secrets. European Commission, Brussels, 4.

https://cordis.europa.eu/article/id/36094-alma-observatory-opens-window-to-unive rses-darkest-secrets

[37] Brown, R.L., Wild, W. and Cunningham, C. (2003) ALMA-The Atacama Large Millimeter Array. Advances in Space Research, 34, 555-559.

https://doi.org/10.1016/j.asr.2003.03.028

[38] Ministerio de Fomento (2008) Instrucción de Hormigón Estructural EHE-08. Spain.

[39] European Committee for Standardization (2002) BS EN 1990. European Union, 119.

[40] ACI Committee (1987) ACI 365.1R-00 Service-Life Prediction. Vol. 15, 292-296. https://doi.org/10.1080/09613218708726837

[41] European Committee for Standardization (2004) EN 1992-1-1 (2004) (English): Eurocode 2: Design of Concrete Structures-Part 1-1: General Rules and Rules for Buildings. Vol. 1, 227.

[42] Barth, F., et al. (2001) Control de la Fisuración en Estructuras de Hormigón Informado por el Comité ACI 224. ACI, 1-53.

[43] Bartels, B., Ermel, U., Sandborn, P. and Pecht, M.G. (2012) Strategies to the Prediction, Mitigation and Management of Product Obsolescence. John Wiley and Sons, Inc., Hoboken. https://doi.org/10.1002/9781118275474

[44] Alfredo Verdoy, S. (2012) La ciencia española ante los recortes. Razon y Fe, 266, 363-368. https://revistas.comillas.edu/index.php/razonyfe/article/view/9911/9311

[45] Mervis, J. (2020) Trump's New Budget Cuts All But a Favored Few Science Programs. Science (80-.), 367, 723-724. https://doi.org/10.1126/science.367.6479.723 